\title{
Tecnoestrés en estudiantes universitarios. Diagnóstico en el marco del covid- 19 en México
}

\section{Technostress in university students. Diagnosis in the framework of covid-19 in Mexico}

María Guadalupe Arredondo-Hidalgo
Universidad de Guanajuato, Guanajuato, México
Diana del Consuelo Caldera-González
Universidad de Guanajuato, Guanajuato, México

\section{Resumen}

Objetivo: Identificar el estrés tecnológico o tecno estrés en estudiantes de Instituciones de Educación Superior (IES) en México, en el primer trimestre de la pandemia del COVID-19. Método: Es un estudio cuantitativo con alcance descriptivo. Se aplicaron 517 encuestas para medir cuatro dimensiones de tecnoestrés. Resultados: De acuerdo con las dimensiones consideradas se obtuvieron las siguientes calificaciones en una escala del 1 al 5: a. Actitudes frente a las TIC: 2.9; b. Estrés escolar: 3.2; c. Efectos en el uso de las TIC: 2.1 ; y d. Redes Sociales y TIC en la Educación: 3.87. Se descubrió que los estudiantes pasan casi 8 horas diarias utilizando TIC para actividades académicas, lo cual excede el tiempo habitual. Discusión y Conclusiones: A causa de la pandemia, el cambio súbito de una educación presencial a una educación a distancia y en línea utilizando diversas TIC, ha generado efectos en los estudiantes como ansiedad, depresión y estrés, por lo cual resulta fundamental la intervención para reducir dichos efectos.

Palabras clave: COVID-19, estudiantes, estrés, tecnología, universidades.

\begin{abstract}
Objective: To identify technological stress or techno stress in students of Higher Education Institutions (HEIs) in Mexico, in the first quarter of the COVID-19 pandemic. Method: It is a quantitative study with descriptive scope. 517 surveys were applied to measure four dimensions of technostress. Results: According to the dimensions considered, the following grades were obtained on a scale from 1 to 5: a. Attitudes towards ICTs: 2.9; b. School stress: 3.2; c. Effects on the use of ICTs: 2.1 ; and d. Social networks and ICTs in Education: 3.87. It was found that students spend almost 8 hours a day using ICT for academic activities, which exceeds the usual time. Discussion and Conclusions: Because of the pandemic, the sudden shift from faceto-face education to distance and online education using various ICTs has generated effects on students such as anxiety, depression and stress, so intervention to reduce these effects is essential.
\end{abstract}

Open Access:

ISSN: $0124-2121$ E-ISSN: $2665-2420$

Editor: Dhayana Fernández Matos TIPOLOGÍA DE ARTÍCULO
Copyright $\odot$ By Educación y Humanismo

Keywords: COVID-19, Students, stress, technology, universities.

Cómo citar este artículo (APA): Arredondo-Hidalgo, M., \& Caldera-González, D. (2022). Tecnoestrés en estudiantes universitarios. Diagnóstico en el marco del covid-19 en México. Educación y Humanismo, 24(42), 90-105. https://doi.org/10.17081/eduhum.24.42.4491 


\section{Introducción}

Sin lugar a duda, las Tecnologías de Información y Comunicación (TIC) han hecho más eficientes diversos procesos, lo cual ha impactado en la vida y la cotidianeidad de las personas en todos los ámbitos.

Respecto al uso de las tecnologías en la educación, Qi (2019) asume que se trata de un arma de dos filos, por un lado, son un aliado para mejorar el rendimiento escolar, pero por el otro, pueden ser detonantes de tensión y estrés.

El objetivo general de esta investigación es identificar el estrés tecnológico en estudiantes de Instituciones de Educación Superior, tanto públicas como privadas, al inicio de la pandemia de COVID-19 en México. Lo anterior, con base en cuatro dimensiones de análisis: 1. Actitud frente a las TIC, 2. Estrés laboral o escolar, 3. Efectos del uso de TIC, y 4. Redes sociales y TIC en la educación.

Se trata de una investigación de tipo cuantitativo con alcance descriptivo y diseño no experimental. El instrumento de recolección de información fue una encuesta de 59 ítems aplicada a una muestra incidental de 517 estudiantes de Instituciones de Educación Superior (IES), tanto públicas, como privadas de México, durante los meses de marzo a mayo de 2020. El periodo de recogida de datos es relevante, debido a que el instrumento se aplicó en el último tercio del curso semestral que comenzó de forma presencial y terminó de manera remota a causa del COVID-19, por lo cual tanto estudiantes como profesores, migraron abruptamente al uso de las TIC en el proceso de enseñanza aprendizaje.

El trabajo se encuentra estructurado en cuatro apartados. En el primero se aborda el marco referencial, donde se define el estrés, el estrés académico, el tecnoestrés y las TIC en la educación. En el segundo se describe la metodología y se da la definición de las dimensiones de análisis. En el tercero se muestran los principales resultados. En el último apartado se dan las conclusiones.

\section{Marco Referencial}

\section{Estrés}

La Organización Internacional del Trabajo (OIT, 2016) afirma que el estrés es un fenómeno natural, propio de las actividades diarias y comunes del siglo 21 . Éste se origina por diversos factores: emocionales, físicos, sociales o económicos, los cuales existen en la vida cotidiana de las personas, quienes reaccionan de diferentes formas para resolver los conflictos que se les presentan de acuerdo con sus entornos personales. 
La American Psychological Association (APA, 2020) indica que el estrés es una experiencia emocional molesta que viene acompañada de cambios bioquímicos, fisiológicos y conductuales predecibles. Se presenta en todas las personas, sin importar edad, género o nivel social y se manifiesta a través de preocupaciones o molestias que afectan la salud tanto física como psicológica. Algunas de las causas del estrés se mencionan en la Figura 1.

\section{Figura 1.}

Causas del estrés



Fuente: elaboración propia con base en OIT (2016)

De acuerdo con datos de la Organización para la Cooperación y el Desarrollo Económicos $(O C D E, 2018)$ en el ámbito del trabajo, la tensión laboral en México estaba en $28.9 \%$, calificación arriba de la media de $27.6 \%$ para los países que conforman el organismo. Esto muestra la intensidad del estrés que se presenta en este entorno.

Por lo anterior, los conceptos de resiliencia y capacidad de adaptación son necesarios para hacer frente a la incertidumbre y que las organizaciones han establecido como parte de los escenarios que deben enfrentar en estos tiempos convulsos. Lo anterior, para ser productivas y competitivas en el contexto internacional.

El ámbito académico no es la excepción. Los distintos actores que participan en IES también sufren de este padecimiento como a continuación lo veremos. 


\section{Estrés académico}

Para el contexto de la educación, Alonso et al. (2009) abordan el término resiliencia en correspondencia con el de vulnerabilidad, afirmando que éstos se manifiestan constantemente en las personas, quienes las confrontan de acuerdo con la influencia del medio en que se desenvuelven. Para Martín (2007) el estrés académico debe considerarse desde las variables moduladoras que son de naturaleza psico-socio-educativa, la cual incorpora el autoconcepto académico, es decir: rendimiento escolar, motivación, salud, bienestar; así como diversas características que emanan de la institución educativa tales como: materias cursadas, exigencia académica, etc.

Dyson y Renk (2006) indican que los estudiantes universitarios generan mayores índices de estrés, dado que deben ajustarse a contenidos de las asignaturas, exámenes o actividades sociales para adaptarse con sus compañeros de clase. Esta perspectiva coincide con las distintas causas de estrés representados en la Figura 1, donde la personalidad en conjunto con los factores del entorno, promueven o inhiben la aparición de este fenómeno.

Pulido et al. (2011) hacen énfasis en que las IES deben observar los niveles de estrés en los estudiantes, dado que éste se relaciona con la depresión y también con la vulnerabilidad académica derivada de los cambios que los jóvenes enfrentan. Vidal et al. (2018) indican tres componentes del estrés académico: estímulos estresores (input), síntomas (indicadores de desequilibrio sistémico) y estrategias de afrontamiento (output). Estos componentes están alineados a las capacidades sociales desde el proceso de enseñanza-aprendizaje que los alumnos desarrollan, tanto con sus compañeros, como con los profesores que son parte esencial de este proceso y quienes afectan directamente en los niveles de exigencia académica (Cabanach et al., 2010).

El estrés académico entonces resulta una constante en los estudiantes, debido a las diversas expectativas internas y externas que se colocan sobre sus hombros (Reddy, Menon \& Thattil, 2018), por lo cual resulta imprescindible identificar las fuentes que lo originan, siendo recientemente una de ellas, el uso intensivo de la tecnología a consecuencia del confinamiento por el COVID-19.

\section{Estrés tecnológico o Tecnoestrés}

Wang, Tan \& Li (2020) afirman que con la tecnología el aprendizaje de los estudiantes universitarios ha mejorado, ya que disfrutan de beneficios sin precedentes, sin embargo, han enfrentado también a mayores demandas, por lo cual han desarrollado estrés tecnológico.

El estrés tecnológico también es conocido como tecnoestrés, término acuñado por Craig Brod en la década de los años 80 en su obra Technostress: the human cost of the computer 
revolution, y la cual a más de 30 años aún se encuentra vigente. De acuerdo con Brod (1984) el tecnoestrés es entendido como una enfermedad que se ocasiona por la falta de habilidades para adaptarse a las nuevas tecnologías.

Cuervo et alı, (2018) lo definen como "el impacto negativo de las actitudes, pensamientos, comportamientos o fisiología corporal causado directa o indirectamente por la tecnología" (p. 19).

Para Alfaro De Prado (2009) el tecnoestrés es:

Una moderna enfermedad de adaptación, causada por la falta de habilidad para tratar con las nuevas tecnologías. La mayor parte de sus síntomas son similares a los del estrés: fatiga, insomnio, depresión, dolores de cabeza y tensiones musculares, aunque el primer síntoma que puede alertar de la presencia del tecnoestrés son episodios de irritabilidad o resistencia obstinada a recibir instrucciones sobre el funcionamiento de cualquier elemento tecnológico (p. 127).

Es posible apreciar en estas aproximaciones que se refiere a una afectación o padecimiento (físico o mental) originado por el uso y/o adaptación a las tecnologías.

El Instituto Nacional de Seguridad y Salud en el Trabajo de España, define el tecnoestrés como "estrés específico derivado de la introducción y uso de nuevas tecnologías en el trabajo, que conlleva efectos psicosociales negativos derivados del uso de las TIC" (INSST, 2020).

Cuervo et al. (2018) coinciden en indicar que el tecnoestrés tiene signos negativos en las conductas de las personas, dada la tensión que imprimen en la dependencia hacia los dispositivos que puedan ser asequibles para el desarrollo de las actividades cotidianas. Entonces, el uso cotidiano de diferentes gadgets. laptops, tabletas o teléfonos inteligentes, ha llevado a una dependencia creciente de aplicaciones que éstos desarrollan como parte de los servicios de telefonía digital. Coppari et al. (2017) aluden a que las generaciones más jóvenes: millenials o centennials, son las más relacionadas con el uso de las TIC y las aplicaciones que han desarrollado para adaptar la vida moderna a estas prácticas; afirman así que los comportamientos de estrés tecnológico se incrementan con el uso de las TIC.

Cuevas (2018) y Prado (2008) coinciden en que el estrés causado por la tecnología lleva a mostrar en las personas, patrones de comportamiento disfuncionales en los lugares de trabajo. Estos pueden presentarse como: falta de concentración, irritabilidad, nula tolerancia hacia los demás, escaso compañerismo, sentimiento de acoso por los superiores, lo que detona la sensación de que las actividades a realizar son una carga que no se puede afrontar. Díaz et al. (2020) indican que este estrés en los jóvenes ocasiona una dependencia hacia la necesidad de tener estos dispositivos en sus actividades diarias, revelando una vulnerabilidad alta, dado que no pueden vivir apartados de su uso. Así también Salanova et al., (2013) aluden al término como technostrain, el cual incide en sentimientos como 
ansiedad, fatiga, escepticismo e ineficacia relacionadas con el uso de las tecnologías.

Por su parte Sanz-Blaz et al. (2019) enfatizan que las redes sociales son parte de la vida cotidiana mayormente en los jóvenes, quienes mientras más información reciben de forma constante, se presentan más ansiosos o estresados en sus actividades, tanto laborales, como académicas.

Ahora bien, como lo mencionan Taylor et al. (2020), durante la pandemia del COVID19, las personas han experimentado una carga extraordinaria de estrés ocasionada por el miedo y la incertidumbre respecto a poder contagiarse y a las consecuencias que se pronostican en materia socioeconómica a nivel mundial.

De esta manera, se plantea esta investigación que busca reconocer los factores de tecnoestrés en los estudiantes universitarios en el inicio de la pandemia del COVID-19, la cual intempestivamente modificó su vida académica.

\section{Las TIC en la Educación}

La Organización de las Naciones Unidas para la Educación, la Ciencia y la Cultura (UNESCO, 2016) afirma que para la educación en la agenda 2030, las TIC tienen un papel esencial en la formación superior de los alumnos. Asimismo, observa que, para América Latina, el reto es el acceso a la educación, es decir que el término de inclusión también se hace presente, dado que la disponibilidad de los recursos es heterogénea. En la actualidad para el acceso a la educación a través de las TIC es necesario un dispositivo, pero también acceso a distintos recursos como un espacio apropiado, electricidad y acceso a internet.

Por lo anterior, podemos afirmar que el tecnoestrés se origina por la falta de competencias tecnológicas, entendidas como conocimientos, habilidades y destrezas para utilizar la tecnología: los tecno recursos, que se refieren a los dispositivos e insumos necesarios para poderlos utilizar como electricidad, acceso a Internet, software y hardware; y también las tecno demandas, que son aquellas exigencias a las cuales es preciso hacerles frente. 


\section{Figura 2.}

Causas del Tecnoestrés

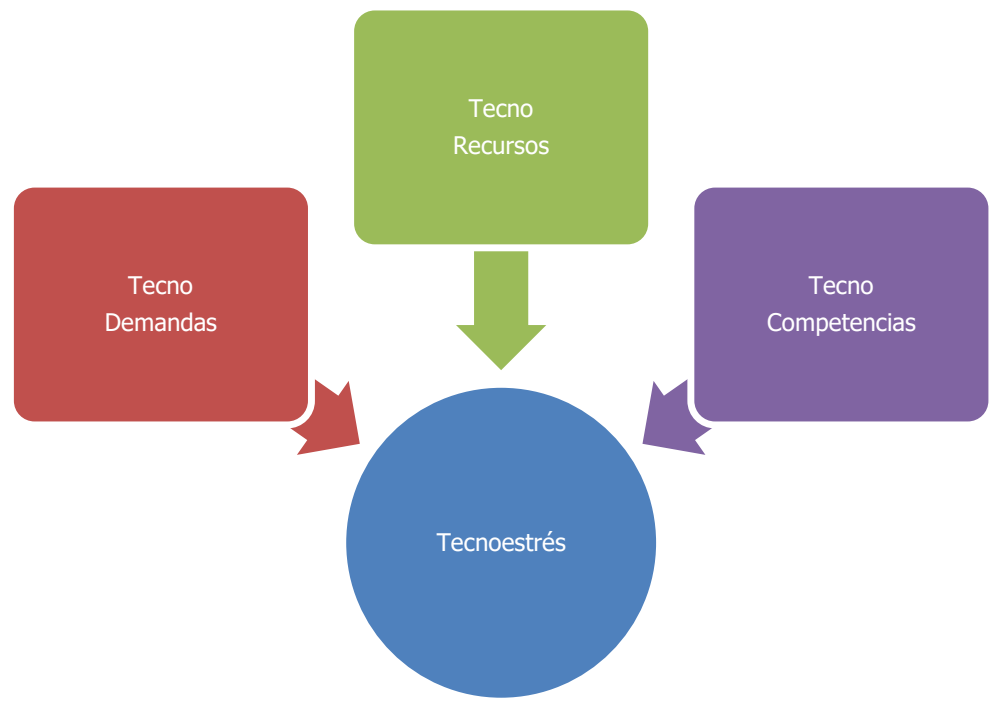

Fuente: elaboración propia (2021)

Así, en concordancia con Coppari et al. (2018) "los efectos del tecnoestrés derivan en una reducción considerable de la calidad de vida, ausentismo laboral y baja producción, así como problemáticas de carácter relacional" (p. 41).

Ahora bien, si nos ubicamos en el ámbito académico universitario, algunas investigaciones mencionan que los efectos del tecnoestrés son ansiedad, tensión, agotamiento, desmotivación y falta de satisfacción, lo cual necesariamente afecta tanto el bienestar de la persona como su rendimiento académico.

Evidentemente con el fenómeno de la pandemia del COVID-19 esto se ha acelerado, generando por un lado el estrés natural a causa del confinamiento con síntomas como la ansiedad y la depresión (Shevlin et al., 2020) y por el otro el estrés que genera el uso intensivo de la tecnología (Molino et al., 2020).

\section{Método}

Esta investigación es de tipo cuantitativo, con enfoque descriptivo. El instrumento aplicado fue una encuesta compuesta por 59 ítems suministrada a través de un servicio de encuestas on line durante el primer semestre de 2020. La escala de Likert fue la siguiente: 
Tabla 1.

Escala de Likert

\begin{tabular}{c|l}
\hline Calificación & \multicolumn{1}{c}{ Descripción } \\
\hline 5 & $\mathrm{CA}=$ Completamente de Acuerdo \\
\hline 4 & $\mathrm{~A}=$ de Acuerdo \\
\hline 3 & $\mathrm{~A} / \mathrm{D}=$ más o menos de acuerdo \\
\hline 2 & $\mathrm{D}=$ en Desacuerdo \\
\hline 1 & $\mathrm{CD}=$ Completamente en Desacuerdo \\
\hline
\end{tabular}

Fuente: elaboración propia (2021)

Se recurrió a una muestra incidental de 517 estudiantes pertenecientes a Instituciones de Educación Superior, tanto públicas como privadas en México. Mayormente, los estudiantes de las IES consideradas para el estudio fueron: Universidad de Guanajuato, Universidad Iberoamericana León, Universidad De La Salle Bajío, Instituto Tecnológico Superior de Guanajuato, así como la Universidad Tecnológica de León; todas ellas ubicadas en la Región Bajío de la República Mexicana.

En la Tabla 2 se describen las dimensiones analizadas y sus definiciones.

Tabla 2.

Dimensiones de análisis

\begin{tabular}{l|l}
\hline \multicolumn{1}{c|}{ Dimensiones } & \multicolumn{1}{c}{ Descripción } \\
\hline 1. Actitud frente a las TIC & $\begin{array}{l}\text { Mide el tiempo en que los estudiantes están inmersos en las } \\
\text { tecnologías, así como la percepción acerca del uso de las TIC. }\end{array}$ \\
\hline 2. Estrés laboral o escolar & $\begin{array}{l}\text { Mide la forma en que los estudiantes reaccionan al uso de las TIC: el } \\
\text { nivel de adopción, desesperación o aburrimiento. }\end{array}$ \\
\hline 3. Efectos del uso de TIC & $\begin{array}{l}\text { Específicamente en el uso de los dispositivos, relacionado a la conexión } \\
\text { o fuera de internet, como navegar, chatear, estar en redes sociales o } \\
\text { viendo video en diferentes plataformas. }\end{array}$ \\
\hline $\begin{array}{l}\text { 4. Redes sociales y TIC en } \\
\text { educación }\end{array}$ & $\begin{array}{l}\text { Medición del aprovechamiento de las redes sociales más usadas, como } \\
\text { herramientas de apoyo en el aprendizaje: WhatsApp, Facebook, } \\
\text { Twitter, YouTube o Instagram. }\end{array}$ \\
\hline
\end{tabular}

Fuente: elaboración propia (2021)

\section{Resultados}

Para la estadística de fiabilidad total, el Alfa de Cronbach para los elementos tipificados fue de 0.864 , lo cual hace que el total de los 59 ítems considerados en el cuestionario, sean adecuados para la investigación realizada.

La Tabla 3, describe los porcentajes para cada una de las 4 dimensiones consideradas en el estudio. 


\section{Tabla 3.}

Alfa de Cronbach por dimensión

\begin{tabular}{c|c|c}
\hline Dimensión & No. ítems & Alfa de Cronbach \\
\hline A. Actitud frente a las TIC & 20 & 0.822 \\
\hline B. Estrés laboral-escolar & 10 & 0.633 \\
\hline C. Efectos del uso de las TIC & 10 & 0.872 \\
\hline D. Redes Sociales y TIC en Educación & 19 & 0.884 \\
\hline
\end{tabular}

Fuente: elaboración propia (2021)

Para el estadístico de fiabilidad, el Alfa de Cronbach más alto corresponde a la dimensión Redes Sociales y TIC en la Educación (0.884), y la más baja fue para la dimensión Estrés laboral-Escolar (0.633). No obstante, la diferencia entre los porcentajes, el segundo índice se considera suficientemente válido para medir la dimensión en la investigación presentada.

La Tabla 4 incorpora los resultados demográficos de las 517 encuestas contestadas.

Tabla 4.

Datos demográficos de la muestra

\begin{tabular}{l|l}
\hline \multicolumn{1}{c|}{ Variables } & \multicolumn{1}{c}{ Resultados } \\
\hline Edad & $81.6 \%$ de 18 a 23 años \\
& $13.5 \%$ de 24 a 25 años \\
& $4.9 \%$ más de 25 años \\
\hline Sexo & $56.8 \%$ Mujeres \\
& $43.2 \%$ Hombres \\
\hline Nivel de escolaridad & Licenciatura: 93\% \\
& Maestría: $7 \%$ \\
\hline
\end{tabular}

Fuente: elaboración propia (2021)

El porcentaje más alto, corresponde a los estudiantes de entre 18 a 23 años, que corresponde al nivel de licenciatura, así también el estudio consideró a alumnos de posgrado, con un $7 \%$ del total de la muestra. 
Figura 3.

Distribución de las áreas del conocimiento

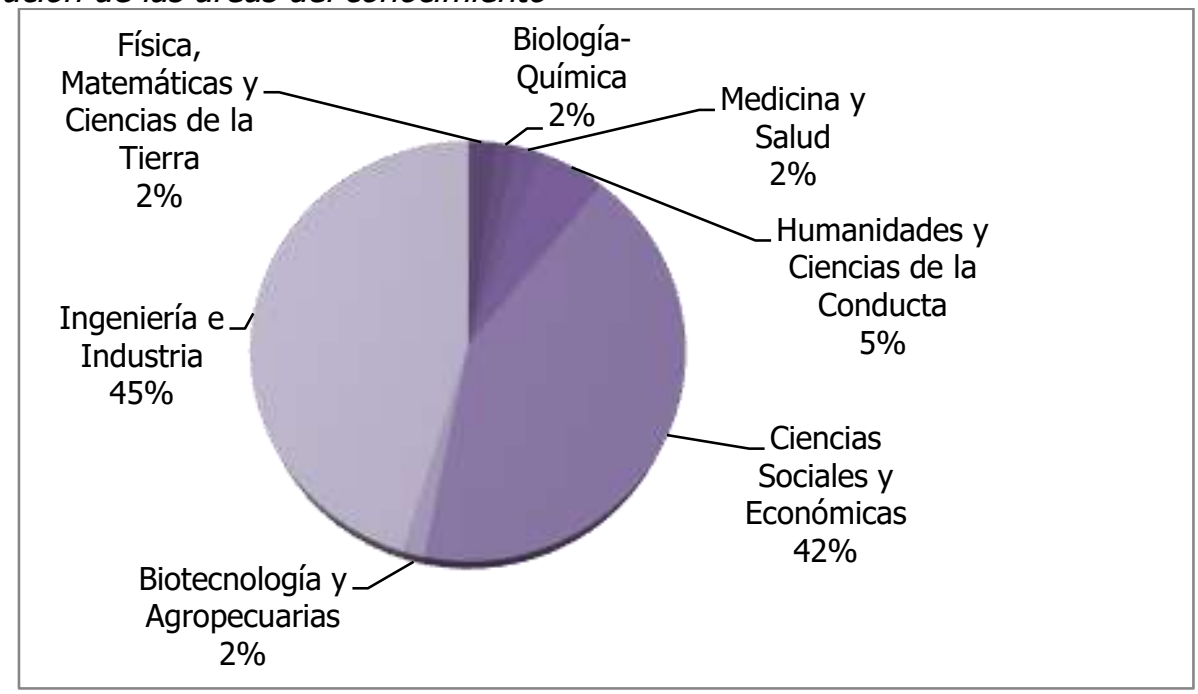

Fuente: elaboración propia (2021)

La Figura 3 muestra que la mayor cantidad de alumnos analizados (45\%) fue del área de Ingeniería e Industria; un porcentaje similar (42\%) fue para el área de Ciencias Económico Administrativas, los demás porcentajes fueron menores para las demás áreas del conocimiento.

Tabla 5.

Cantidad de horas usadas en las TIC

\begin{tabular}{c|c|c|c}
\hline Tiempo & Frecuencia & Porcentaje & Porcentaje acumulado \\
\hline Menos de 1 hora & 6 & 1.2 & 1.2 \\
\hline Entre 1 y 4 hrs al día & 93 & 18.0 & 19.1 \\
\hline Entre 5 y 8 hrs al día & 224 & 43.3 & 62.5 \\
\hline Entre 9 y 12 hrs al día & 144 & 27.9 & 90.3 \\
\hline Más de 12 hrs al día & 50 & 9.7 & 100.0 \\
\hline Total & 517 & 100.0 & \\
\hline
\end{tabular}

Fuente: elaboración propia (2021)

Respecto a las horas invertidas en el uso de las TIC, la Tabla 3 indica que en primer lugar destacan los estudiantes que emplean entre 5 y 8 horas diarias (43.3\%); en segundo lugar, están los estudiantes que pasan entre 9 y 12 horas en el desarrollo de sus actividades académicas (casi 28\%). Como puede apreciarse, el primer porcentaje es realmente alto, considerando que, en forma presencial, las clases diarias son en promedio de 6 horas diarias, para una carga académica regular en las IES. Con base en el análisis, es posible afirmar que la distribución de frecuencias en la medición del tiempo es normal, lo cual significa que los estudiantes están dedicando pocas o nulas horas al trabajo autónomo o independiente, fuera de aula. 
Tabla 6.

Estadísticos de resumen de las dimensiones

\begin{tabular}{c|c|c|c|c}
\hline Dimensiones & Media & Mínimo & Máximo & Ítems \\
\hline 1. Actitud frente a las TIC & 2.910 & 1.812 & 4.125 & 20 \\
\hline 2. Estrés laboral-Escolar & 3.221 & 1.871 & 4.376 & 10 \\
\hline 3. Efectos del uso de las TIC & 2.175 & 1.585 & 3.386 & 10 \\
\hline 4. Redes Sociales y TIC en la Educación & 3.871 & 2.787 & 4.282 & 19 \\
\hline
\end{tabular}

Fuente: elaboración propia (2021)

La Tabla 6 indica la calificación que los estudiantes han dado a las 4 dimensiones analizadas.

Para la primera dimensión de Actitudes frente a las TIC, los estudiantes presentan un 2.9 de 5 lo que significa que tienen una actitud negativa o de rechazo hacia las TIC. Esta respuesta se muestra muy polarizada, es decir hay quienes aceptan y se sienten muy cómodos usando las TIC y otros, que no se sienten identificados de manera alguna con este tipo de herramientas. Podemos explicar este comportamiento ya que generalmente las actividades académicas se llevaban a cabo de manera presencial y no intermediaba la tecnología en el proceso, por lo cual el cambio súbito y obligado, generó un sentido de rechazo hacia las TIC.

Para la segunda dimensión que es Estrés escolar, se presenta una tensión más representativa en los alumnos, dado que la calificación fue de 3.2, lo cual indica un estrés que se origina desde la misma actividad académica, es decir, la demanda por un buen desempeño tanto por el mismo individuo como por su entorno de referencia; demanda que se tensiona por la incorporación de las TIC, ya que no necesariamente el estudiante está habituado a utilizarlas en su proceso de enseñanza aprendizaje.

El índice de los Efectos en el uso de las TIC, que es la tercera dimensión, fue el más bajo en la calificación total con 2.1, lo cual da cuenta de la existencia de consecuencias percibidas como negativas por los propios estudiantes. Ansiedad es de las manifestaciones más recurrentes.

La cuarta dimensión que es Redes Sociales y TIC en la Educación obtuvo una calificación de 3.87, lo cual puede referirse a que los estudiantes están más habituados a utilizar las Redes Sociales en su vida habitual, lo cual facilitó su aprovechamiento como herramienta de apoyo en el aprendizaje y también para la comunicación entre sus pares para hacer más ameno el proceso. 


\section{Discusiones y conclusiones}

Como se ha mencionado, el término de tecnoestrés no es nuevo, Picón, Toledo y Navarro (2017) indican que, en la década de los ochenta, fue definido por Craig Brod, y en cuyo concepto se basaron para definir las variables que lo originan, alineando otros conceptos como: la tecno ansiedad o la tecno adicción. Desde que el uso de la computadora y otras tecnologías irrumpieron el mundo cotidiano, se han generado impactos diversos a nivel individual, lo cual implica procesos de adaptación y también de aprendizaje.

Entendido como los efectos negativos que causa el uso de la tecnología en el ser humano, el tecnoestrés representa una enfermedad muy común en nuestro tiempo, más aún, cuando a causa de una pandemia a nivel global, el trabajo se lleva a cabo a través del uso intensivo de la tecnología.

En el ámbito académico el estrés de por sí genera efectos como depresión, ansiedad, problemas de conducta, irritabilidad, entre otros, llegando inclusive a generar incapacidad para concentrarse, miedo al fracaso, percepción negativa del futuro, propensión a mayores conductas de riesgo, como consumo de alcohol y drogas, actividades sexuales sin protección, inactividad física, malos patrones de alimentación y sueño, inclusive algo más grave, la presión que algunos estudiantes enfrentan para obtener un buen rendimiento es tan severa que aumenta cinco veces más la propensión al suicidio (Reddy, Menon \& Thattil, 2018).

La investigación desarrollada fue una primera aproximación para medir el tecnoestrés en estudiantes universitarios a partir de cuatro dimensiones, descubriéndose que, el cambio súbito a causa del COVID-19, de una educación presencial a una educación remota y en línea utilizando diversas TIC, ha generado en ellos consecuencias negativas como ansiedad, depresión y estrés, lo cual se percibe en dos de las dimensiones analizadas: Actitud frente a las TIC y Efectos en el uso de las TIC.

Así como lo consideran Toribio y Franco (2016), resulta esencial reconocer los estresores académicos más influyentes para poder mejorar tanto el bienestar de los estudiantes como su rendimiento académico y justamente en nuestra investigación encontramos síntomas de ansiedad, tensión, agotamiento, desmotivación y falta de satisfacción, todo lo cual se asocia al uso de la tecnología y también al miedo y la incertidumbre ocasionados por la pandemia.

Los resultados de la investigación indican que los estudiantes se abstraen en las clases de forma sincrónica, tratando de resolver sus actividades en el momento de la clase a distancia, sin embargo, tienen múltiples distractores desde casa, donde generalmente están tomando sus clases. Los estudiantes no están dedicando horas al trabajo independiente, porque se muestran aburridos y desean no tener más trabajo fuera del horario escolar, ya que esto les genera mayor estrés. Pese a lo anterior, los estudiantes se encuentran agotados ya que manifiestan que tienen que estar concentrados en las clases a distancia. Aunado a lo anterior, también encontramos problemas respecto a la brecha tecnológica tanto en la 
falta de habilidades tecnológicas como en la falta de recursos para hacerse de la tecnología, es decir, que la pandemia ha evidenciado la desigualdad existente entre los estudiantes, ya que algunos no tienen problemas para contar con dispositivos y acceso a internet, y hay otros quienes, incluso, han abandonado los estudios porque no cuentan con ello, lo cual les genera frustración y estrés.

Algunos de los conceptos con los que relacionamos el tecnoestrés son tecno ansiedad, tecno fatiga, tecno invasión, tecno adicción, tecno complejidad e inclusive sobrecarga tecnológica (Saavedra, 2007; Brivio et al., 2018), y como sus nombres lo indican, se refieren a los factores de riesgo psicosocial derivados del uso intensivo de la tecnología.

En este orden de ideas, resulta imprescindible considerar, como lo apuntan Villavicencio, Ibarra y Calleja (2020), que "las consecuencias de sufrir tecnoestrés impactan tanto a nivel personal (fisiológico-psicosocial), a nivel profesional (como estudiante), y a nivel laboral (como trabajador en las organizaciones), surgiendo así un tema relevante a estudiar" (p. 18).

El reto entonces es grande para toda una sociedad global que sigue sumida en la incertidumbre a causa del COVID-19, pandemia que a mediados del año 2020 ha causado cerca de 845,000 muertes y más de 25 millones de casos a nivel mundial (WHO, 2020).

Las TIC pueden crear exigencias para las personas que, en ocasiones, son incapaces de asumir, bien sea por falta de formación, adaptación o por el uso inadecuado de las mismas (Aragüez, 2017). En este sentido, las IES deben reinventarse para continuar con su misión, pero a la vez con la detección y disminución de los factores de riesgo psicosocial para los estudiantes a causa del seguimiento de sus clases de manera remota y virtual y necesariamente a causa de haber vivido uno de los momentos más importantes de la época actual: la pandemia ocasionada por el COVID-19.

Indudablemente el reto es grande, sin embargo, ya existen algunas propuestas que hablan del término Tecnologías Positivas (Brivio et al., 2018), las cuales permitan prevenir el tecnoestrés y promover experiencias positivas y el bienestar general a través de una cultura de seguridad organizacional efectiva.

Dado que se trata de una investigación inicial, para estudios futuros, podría tomarse la base de este trabajo, y reunir datos de una muestra más amplia. Lo anterior, para considerar momentos clave de intervención en las instituciones de educación superior con el objetivo de diseñar y generar estrategias para el beneficio, tanto de los estudiantes, como de los docentes que son parte fundamental del proceso enseñanza-aprendizaje. 


\section{Referencias}

Alfaro De Prado, A. (2009). Estrés Tecnológico: Medidas Preventivas para Potenciar La Calidad de Vida Laboral. Temas Laborales, (10), 123-155.

Alonso, E., Valdés, A., Cabral, F. y Galván, L. (2009). Vulnerabilidad al Estrés en Estudiantes del Instituto Tecnológico Superior de Cajeme (Itesca). INED. Universidad Pedagógica de Durango, (11), 60-76.

American Psychological Association [APA]. (2020). Comprendiendo el Estrés Crónico. APA. https://WwW.apa.org/centrodeapoyo/estres-cronico

Aragüez, L. (2017). El impacto de las tecnologías de la información y de la comunicación en la salud de los trabajadores: el tecnoestrés. E-Revista Internacional de la protección Social, 2(2), 169-190.

Brivio, E., Gaudioso, F., Vergine, I., Mirizzi, C. R., Reina, C., Stellari, A., \& Galimberti, C. (2018). Preventing Technostress Through Positive Technology. Frontiers in psychology, (9), 2569.

Brod, C. (1984). Technostress: The human cost of the computer revolution. Addison-Wesley.

Cabanach, R., Fernández-Cervantes, R., González, L. y Freire, C. (2010). Estresores académicos percibidos por estudiantes universitarios de ciencias de la salud. Fisioterapia, 32(4), 151-158.

Coppari, N., Bagnoli, L., Codas, G., Martínez, M., Martínez, U. y López, H. (2017). Uso de Tecnologías de la Comunicación e Información y Tecnoestrés en Estudiantes Paraguayos: su relación con la edad. Cuadernos de Neuropsicología, 11(3), 166-181.

Coppari, N., Bagnoli, L., Codas, G., López, H., Martínez, L., y Martínez, M. (2018). Validez y confiabilidad del cuestionario de tecnoestrés en estudiantes paraguayos. Perspectivas en Psicología, 15(2), 40-55.

Cuervo Carabel, T., Orviz Martínez, N., Arce García, S., \& Fernández Suárez, I. (2018). Tecnoestrés en la Sociedad de la Tecnología y la Comunicación: revisión bibliográfica a partir de la Web of Science. Archivos de Prevención de Riesgos Laborales, 21(1), 18-25. https://dx.doi.org/10.12961/aprl.2018.21.01.4

Cuevas, C. (2018). Algunos antecedentes conceptuales acerca del síndrome tecno estrés del capital humano en las organizaciones. Revista ciencia administrativa, (6), 142-158.

Díaz-López, A., Maquilón-Sánchez, J., Mirete-Ruíz, A. (2020). Uso desadaptativo de las TIC en adolescentes: perfiles, supervisión y estrés tecnológico. Comunicar. Revista Científica de Comunicación y Educación, 64(28), 29-38. 


\section{https://doi.org/10.3916/C64-2020-03}

Dyson, R. \& Renk, K. (2006). Freshmen adaptation to university life: depressive symptoms, stress, and coping. Journal of Clinical Psychology, 62(10), 1231-1244.

Martín, I. (2007). Estrés académico en Estudiantes Universitarios. Revista Apuntes de Psicología, 25(1), 87-99.

Molino, M., Ingusci, E., Signore, F., Manuti, A., Giancaspro, M. L., Russo, V., \& Cortese, C. G. (2020). Wellbeing Costs of Technology Use during Covid-19 Remote Working: An Investigation Using the Italian Translation of the Technostress Creators Scale. Sustainability, 12(15), 5911.

Organización de las Naciones Unidas para la Educación, la Ciencia y la Cultura [UNESCO]. (2016). Tercer Estudio Regional Comparativo y Explicativo (TERCE). UNESCO. http://www.unesco.org/new/es/media-services/single-view-tvrelease/news/tercer_estudio_regional_comparativo_y_explicativo_terce_a/

Organización Internacional del Trabajo [OIT] (2016). Estrés Laboral. OIT. https://www.ilo.org/wcmsp5/groups/public/---americas/---rolima/documents/genericdocument/wcms_475146.pdf

Organización para la Cooperación y el Desarrollo Económicos [OCDE] (2018). La nueva Estrategia de empleo de la OCDE. Situación para México. OCDE. https://www.oecd.org/mexico/jobs-strategy-MEXICO-ES.pdf

Picón, C., Toledo, S., \& Navarro, V. (2017). Tecnoestrés: Identificación y prevalencia en el personal docente de la Facultad de Medicina de la Universidad Nacional del Nordeste. Revista de la Facultad de Medicina de la Universidad Nacional del Nordeste, 36(3), 41-51.

Prado-Sagrera, A. (2008). Nuevas tecnologías y nuevos riesgos laborales: estrés y tecnoestrés. Revista digital de salud y seguridad en el trabajo, (1), 1-23.

Pulido, M., Serrano, M., Valdés, E., Chávez, M., Hidalgo, P. y Vera, F. (2011). Estrés académico en estudiantes universitarios. Psicología y Salud, 1(21), 31-37.

Qi, C. (2019). A double-edged sword? Exploring the impact of students' academic usage of mobile devices on technostress and academic performance. Behaviour \& Information Technology, 38(12), 1337-1354. https://doi.org/10.1111/isj.12169

Reddy, K. J., Menon, K. R., \& Thattil, A. (2018). Academic stress and its sources among University students. Biomedical and Pharmacology Journal, 11(1), 531-537.

Salanova, M., Llorens, S., \& Cifre, E. (2013). The dark side of technologies: Technostress 
among users of information and communication technologies. International Journal of Psychology, 48(3), 422-436. https://doi-org.erevistas.ugto. $\mathrm{mx} / 10.1080 / 00207594.2012 .680460$

Sanz-Blas, S., Buzova, D. \& Miquel-Romero, M.J. (2019). From Instagram overuse to instastress and emotional fatigue: the mediation of addiction. Emerald Insight, (2), 143-161.

Shevlin, M., McBride, O., Murphy, J., Miller, J. G., Hartman, T. K., Levita, L., \& Bennett, K. M. (2020). Anxiety, Depression, Traumatic Stress, and COVID-19 Related Anxiety in the UK General Population during the COVID-19 Pandemic. Working Draft.

Taylor, S., Landry, C., Paluszek, M., Fergus, T. A., McKay, D., \& Asmundson, G. J. (2020). Development and initial validation of the COVID Stress Scales. Journal of Anxiety Disorders, (72), 1-7.

Toribio-Ferrer, C., y Franco-Bárcenas, S. (2016). Estrés académico: el enemigo silencioso del estudiante. Revista Salud y Administración, 3(7), 11-18.

Vidal, Conti, J., Muntaner-Mas, A., y Palou Sampol, P. (2018). Diferencias de estrés y afrontamiento del mismo según el género y cómo afecta al rendimiento académico en estudiantes universitarios. Contextos Educativos. Revista de Educación, (22), 181-195.

Villavicencio-Ayub, E., Aguilar, D. G. I., \& Calleja, N. (2020). Tecnoestrés en población mexicana y su relación con variables sociodemográficas y laborales. Psicogente, 23(44), 1-27.

Wang, X., Tan, S. C., \& Li, L. (2020). Measuring university students' technostress in technology-enhanced learning: Scale development and validation. Australasian Journal of Educational Technology, 36(4), 96-112.

World Health Organization [WHO] (2020). Coronavirus disease (COVID-19) Weekly Epidemiological Update and Weekly Operational Update. WHO. https://www.who.int/emergencies/diseases/novel-coronavirus-2019/situationreports/ 